\title{
Mortalidad perioperatoria y volumen quirúrgico de colecistectomías en el régimen contributivo en Colombia
}

\author{
Perioperative mortality and surgical volume of cholecystectomies in \\ the contributory regime in Colombia
}

\author{
Daniela Escalante-Arbeláez $\mathbb{D}^{\mathbb{D}}$, Marcela Bernal-Gutiérrez² $\mathbb{D}$, \\ Giancarlo Buitrago-Gutiérrez ${ }^{3}$ D
}

1 Estudiante de Medicina, Semillero de investigación clínica, Universidad Nacional de Colombia, Bogotá, D.C., Colombia.
Estudiante de Medicina, Semillero de investigación clínica, Instituto de investigaciones clínicas, Departamento de cirugía, Univer-
sidad Nacional de Colombia, Bogotá, D.C., Colombia.
Profesor asociado, Universidad Nacional de Colombia, Instituto de investigaciones clínicas, Departamento de cirugía, Universidad
Nacional de Colombia, Hospital Universitario Nacional de Colombia, Bogotá, D.C., Colombia.

Trabajo presentado en III Jornada de Investigación, Facultad de Medicina, Universidad Nacional de Colombia. Bogotá D.C., 20 de noviembre de 2019.

\section{Resumen}

Introducción. La patología biliar es una causa frecuente de intervención quirúrgica, pero en Colombia no se cuenta con datos oficiales de mortalidad ni volumen quirúrgico asociados a este procedimiento.

El objetivo de este estudio fue determinar el volumen de colecistectomías y describir la tasa general de mortalidad perioperatoria en seis regiones geográficas del país, en el periodo de 2012 a 2016.

Métodos. Estudio de cohortes retrospectivo que incluyó pacientes mayores de 18 años, afiliados al régimen contributivo de salud, llevados a colecistectomía. Se utilizó la base de datos de suficiencia de la Unidad de Pago por Capitación (UPC), la base de estadísticas vitales del Departamento Administrativo Nacional de Estadística (DANE) y la del Sistema Integrado de Información de la Protección Social (SISPRO).

Resultados. Durante el periodo de estudio, se realizaron 192.080 colecistectomías, lo que corresponde a 206 colecistectomías por 100.000 habitantes. Se encontró mayor volumen quirúrgico en personas entre los 65 y 80 años, en el sexo femenino y en la ciudad de Bogotá. La mortalidad perioperatoria encontrada correspondió al 0,6 \%, siendo mayor en los pacientes de sexo masculino, de edad avanzada, en aquellos en quienes se practicó laparotomía y en los pacientes operados en la ciudad de Bogotá.

Fecha de recibido: 21/05/2020 - Fecha de aceptación: 12/06/2020

Correspondencia: Marcela Bernal-Gutiérrez, Calle 67D No 59 - 65, Torre 1, Apto 102, Bogotá, D.C., Colombia. Teléfono: 3505148122. Correo electrónico: gimbernalgu@unal.edu.co

Citar como: Escalante-Arbeláez D, Bernal-Gutiérrez M, Buitrago-Gutiérrez. Mortalidad perioperatoria y volumen quirúrgico de colecistectomías en el régimen contributivo en Colombia. Rev Colomb Cir. 2021;36:83-90.

https://doi.org/10.30944/20117582.705

Este es un artículo de acceso abierto bajo una Licencia Creative Commons - BY-NC-ND https://creativecommons.org/licenses/by-ncnd/4.0/deed.es 
Discusión. De acuerdo con los hallazgos, esta investigación puede servir de base para estudios posteriores, que muestren claramente las cifras oficiales de volumen quirúrgico y de mortalidad por colecistectomía en Colombia.

Palabras clave: colecistectomía; volumen quirúrgico; perioperatorio; tasa de mortalidad; régimen contributivo.

\begin{abstract}
Introduction. Biliary pathology is a frequent cause of surgical intervention, but in Colombia there are no official data on mortality or surgical volume associated with this procedure. The objective of this study is to determine the volume of cholecystectomies and to describe the general perioperative mortality rate in six geographic regions during the period from 2012 to 2016.

Methods. Retrospective cohort study that included patients older than 18 years and affiliated to the contributory health regimen who underwent cholecystectomy. The sufficiency database of the Capitation Payment Unit (UPC), the vital statistics database of the National Administrative Department of Statistics (DANE) and that of the Integrated Social Protection Information System (SISPRO) were used.

Results. During the study period, 192,080 cholecystectomies were performed, corresponding to 206 cholecystectomies per 100,000 inhabitants. Higher surgical volume was found in people between 65 and 80 years old, in females and geographically in the city of Bogotá. The perioperative mortality found corresponded to $0.58 \%$, being higher in elderly male patients, in those who underwent laparotomy and in patients operated on in the city of Bogotá.
\end{abstract}

Discussions. According to the findings, these results can serve as the basis for subsequent studies that clearly show the official figures for surgical volume and mortality from cholecystectomy in Colombia.

Keywords: cholecystectomy; surgical volume; mortality rate; preoperatory; contributive regime.

\section{Introducción}

La colecistectomía, uno de los procedimientos más realizados alrededor del mundo por los cirujanos generales, es el tratamiento quirúrgico de elección para la colelitiasis y la colecistitis, y se asocia con un bajo riesgo de eventos adversos mayores 1. A pesar de su baja incidencia, la mortalidad perioperatoria asociada a colecistectomía ha sido ampliamente estudiada por considerarse menos aceptable que la ocurrida en procedimientos quirúrgicos de alto riesgo ${ }^{1,2}$. La tasa de letalidad atribuida a condiciones prevalentes y de tratamiento conocido, como la colelitiasis o la colecistitis, se incrementa cuando no hay acceso a cuidado quirúrgico oportuno, como se ha observado en algunas regiones de ingresos bajos o medios ${ }^{3}$.

A partir de 1950, y con la introducción de la técnica laparoscópica, el volumen de colecistectomías ha venido aumentando significativamente. En Estados Unidos se practican 700.000 colecis- tectomías al año, lo que representa un porcentaje significativo de los gastos en salud en ese país, estimado en 6,5 billones de dólares anuales ${ }^{4}$.

En Colombia, se espera actualmente la cobertura nacional para ofrecer este procedimiento quirúrgico, ya que todos los departamentos cuentan con al menos un hospital de mediana complejidad ${ }^{5}$, sin embargo, se desconoce el volumen quirúrgico de colecistectomías a nivel nacional. No hay datos oficiales acerca de la mortalidad perioperatoria asociada a este procedimiento, y menos aún de los factores de riesgo relacionados con mortalidad o su relación con características poblacionales como sexo, edad o distribución geográfica.

En 2015, la Lancet Commission on Global Surgery, atendiendo a las necesidades de la nueva era de salud global, y centrándose en la atención quirúrgica como componente integral de los cuidados en salud, y lo que su inversión representa para la economía de un país, propuso unas metas entre 
las que se encuentran: un mínimo de 5000 procedimientos por 100.000 habitantes para 2030, rastrear la mortalidad perioperatoria en el $80 \%$ de los países para 2020 y así establecer metas nacionales para 2030 , acceso temprano a tratamiento quirúrgico, y 20 especialistas por 100.000 habitantes para 2030, entre otros ${ }^{3}$.

El objetivo de este estudio fue establecer el volumen de colecistectomías por año a nivel nacional, y comparar estos datos por grupo etario, región y sexo. Además, describir la tasa de mortalidad perioperatoria en pacientes llevados a colecistectomía y comparar las tasas específicas por edad en seis regiones geográficas, en adultos pertenecientes al régimen contributivo de salud, que fueron llevados a colecistectomía entre 2012 y 2016.

\section{Métodos}

\section{Demografía poblacional y diseño del estudio}

Estudio de cohortes retrospectivo, en el que se incluyeron pacientes mayores de 18 años que fueron sometidos a colecistectomía entre el 1 de enero de 2012 y el 31 de diciembre de 2016, y que se encontraban afiliados al régimen contributivo de salud. Para el desarrollo del estudio se utilizaron tres bases de datos oficiales: la base de datos de suficiencia de la Unidad de Pago por Capitación (UPC) ${ }^{1}$, en la que se encontraron registros de las colecistectomías realizadas en todo el país mediante los códigos de Clasificación Única de Procedimientos en Salud (CUPS), motivo por el cual no se diferencia entre procedimientos de urgencia o programados. La base de datos de estadísticas vitales del DANE, donde se pudo acceder a la identificación anonimizada de las personas fallecidas durante el tiempo del estudio, y por último la base de datos del Sistema Integrado de Información de la Protección Social (SISPRO), que contenía el número de afiliados por las aseguradoras o Empresas Promotoras de Salud (EPS) del régimen contributivo a nivel nacional. Se utilizó Stata/MP 14.2 para el procesamiento y análisis estadístico de las bases de datos. El protocolo del presente estudio fue evaluado y aprobado por el comité de ética de la Facultad de Medicina de la Universidad Nacional de Colombia. El presente estudio se clasifica en la categoría "sin riesgo", de acuerdo con lo establecido en la Resolución 8430 de 1993.

\section{Variables}

Se describió el número de colecistectomías realizadas en todo el país, se calculó la mortalidad general a 30 días con su respectiva tasa y la mortalidad específica por edad. Se estimó el volumen quirúrgico teniendo en cuenta los afiliados a EPS del régimen contributivo, así como el número de procedimientos por 100.000 habitantes (afiliados en este caso).

Las variables identificadas en este estudio fueron: sexo, grupo etario, tipo de procedimiento y región geográfica: Atlántica, Bogotá, Central, Oriental, Pacífica, otras. En "otras", se integraron departamentos que no se pudieron incluir en las primeras cuatro regiones tales como: Amazonas, Arauca, Casanare, Guainía, Guaviare, Putumayo, San Andrés, Vaupés y Vichada, así como las personas en custodia por parte del Instituto Nacional Penitenciario y Carcelario (INPEC).

\section{Análisis estadístico}

Una vez procesada y organizada la información de las tres bases de datos en Stata/MP 14.2, se estimaron las colecistectomías realizadas de 2012 a 2016, comparando el tipo de procedimiento con sexo, grupo etario y región geográfica. Una vez obtenida esta información se determinó la mortalidad durante los 30 días posteriores a la cirugía, utilizando la diferencia entre la fecha de la cirugía registrada en la base de datos de suficiencia de la UPC y la fecha de defunción registrada en la base de estadísticas vitales del DANE para el mismo paciente, cuya identificación anonimizada estaba en las dos bases de datos. Se agrupó la mortalidad por región y grupo etario para el cálculo de tasa específica de mortalidad. Todas las tasas fueron estimadas por 1000 cirugías.

Se estimaron los procedimientos realizados por EPS de 2012 a 2016, utilizando la base de datos de suficiencia de la UPC. Por otro lado, se de- 
terminó el número de afiliados en las mismas EPS también registradas en la base de datos de SISPRO. Para calcular el volumen quirúrgico se dividieron los procedimientos realizados sobre el número de afiliados. Se comparó el año de realización del procedimiento con grupo etario y sexo, y de igual forma la región geográfica con el año de la cirugía y el grupo etario. El volumen quirúrgico se estimó por 100.000 personas afiliadas a EPS del régimen contributivo.

\section{Resultados}

El estudio incluyó 192.002 personas que fueron llevadas a colecistectomía durante el periodo de estudio. En cuanto a las características demográficas de la población incluida en el estudio, la mayoría de las personas operadas estaban entre los 18 y los 49 años de edad, eran residentes en Bogotá, D.C. Además, se encontró que el número de personas a quienes se les realizó colecistectomía laparoscópica fue más de seis veces mayor a las personas con colecistectomía abierta o por laparotomía (tabla 1).

Al comparar el tipo de procedimiento con grupo etario y la ubicación geográfica, se observó que a las personas jóvenes se les realizó más colecistectomías por laparoscopia, así como a las que se ubicaron en la zona central del país, incluyendo Bogotá, D.C.

La tasa de mortalidad perioperatoria fue mayor en hombres, en personas mayores de 80 años y en la región oriental (tabla 2). Al comparar la mortalidad con el tipo de procedimiento, se encontró que la tasa de mortalidad fue mayor en quienes se operaron por vía abierta y eran mayores de 80 años, y en quienes se operaron en la ciudad de Bogotá por esta misma vía.

Los pacientes menores de 50 años ubicados en la zona oriental, presentaron mayor tasa de mortalidad, así como los mayores de 80 años operados en la zona atlántica (tabla 3). En todos los grupos etarios se observó mayor mortalidad en hombres que en mujeres (tabla 4).

El volumen quirúrgico total para los 5 años de estudio fue de 206 por 100.000 habitantes. Se presentó mayor volumen quirúrgico en mujeres que en hombres, se realizaron más colecistectomías en mayores de 65 años y, geográficamente, en la ciudad de Bogotá, D.C. En 2013 se presentó el pico de volumen quirúrgico, en contraste con 2015, donde la cifra disminuyó (tabla 5). La región Pacífica presentó el mayor volumen quirúrgico en 2015, en contraste con los otros años en los que predominó en Bogotá, D.C.

Tabla 1. Características sociodemográficas de los pacientes incluidos.

\begin{tabular}{|c|c|c|c|c|}
\hline Variables & & $\begin{array}{c}\text { Colecistectomía } \\
\text { abierta } \\
n=25.542 \\
n(\%)\end{array}$ & $\begin{array}{c}\text { Colecistectomía } \\
\text { laparoscópica } \\
n=166.460 \\
n(\%)\end{array}$ & $\begin{array}{c}\text { Total } \\
\mathrm{n}=192.002 \\
\mathrm{n}(\%)\end{array}$ \\
\hline \multirow[t]{2}{*}{ Sexo } & Masculino & $9016(16,6)$ & $45.361(83,4)$ & 54.377 \\
\hline & Femenino & $16.526(12)$ & $121.099(88)$ & 137.625 \\
\hline \multirow[t]{4}{*}{ Grupo etario (años) } & $18-49$ & $11.654(11,37)$ & $90.772(88,62)$ & 102.426 \\
\hline & $50-64$ & $7365(13,73)$ & $46.254(86,26)$ & 53.619 \\
\hline & $65-80$ & $4876(17,66)$ & $22.729(82,33)$ & 27.605 \\
\hline & $>80$ & $1647(19,71)$ & $6705(80,28)$ & 8352 \\
\hline \multirow[t]{6}{*}{ Región geográfica } & Atlántica & $4042(20,04)$ & $16.119(79,95)$ & 20.161 \\
\hline & Bogotá D.C. & $4488(7,16)$ & $58.146(92,83)$ & 62.634 \\
\hline & Central & $7118(14,48)$ & $42.008(85,51)$ & 49.126 \\
\hline & Oriental & $6675(23,80)$ & $21.363(76,19)$ & 28.038 \\
\hline & Pacífica & $2752(9,11)$ & $27.456(90,88)$ & 30.208 \\
\hline & Otros departamentos & $467(25,44)$ & $1368(74,55)$ & 1835 \\
\hline Total & & $25.542(13,30)$ & $166.460(86,69)$ & 192.002 \\
\hline
\end{tabular}


Tabla 2. Mortalidad perioperatoria de la colecistectomía, caracterizada por tipo de procedimiento.

\begin{tabular}{|c|c|c|c|c|}
\hline \multicolumn{2}{|l|}{ Variables } & $\begin{array}{c}\text { Colecistectomía } \\
\text { abierta }\end{array}$ & $\begin{array}{l}\text { Colecistectomía } \\
\text { laparoscópica }\end{array}$ & Total \\
\hline & & \multicolumn{3}{|c|}{ Muertes/Total de pacientes operados (Tasa por 1000 cirugías) } \\
\hline \multirow[t]{2}{*}{ Sexo } & Masculino & $266 / 9016(29,50)$ & $210 / 45.361(4,62)$ & $476 / 54.377(8,75)$ \\
\hline & Femenino & $254 / 16.526(15,36)$ & $273 / 121.099(2,25)$ & $527 / 137.625(3,82)$ \\
\hline \multirow[t]{4}{*}{ Grupo etario } & $18-49$ & $26 / 11.654(2,23)$ & $30 / 90.772(0,33)$ & $56 / 102.426(0,54)$ \\
\hline & $50-64$ & $91 / 7365(12,35)$ & $84 / 46.254(1,81)$ & $175 / 53.619(3,26)$ \\
\hline & $65-80$ & $204 / 4876(41,83)$ & $190 / 22.729(8,35)$ & $394 / 27.605(14,27)$ \\
\hline & $>80$ & $199 / 1647(120,82)$ & $179 / 6705(26,69)$ & $378 / 8352(45,25)$ \\
\hline \multirow[t]{7}{*}{ Región geográfica } & Atlántica & $54 / 4042(13,35)$ & $33 / 16.119(2,04)$ & $87 / 20.161(4,31)$ \\
\hline & Bogotá D.C. & $143 / 4488(31,86)$ & $174 / 58.146(2,99)$ & $317 / 62.634(5,06)$ \\
\hline & Central & $156 / 7118(21,91)$ & $109 / 42.008(2,59)$ & 265/49.126 (5,39) \\
\hline & Oriental & $92 / 6675(13,78)$ & $66 / 21.363(3,08)$ & $158 / 28.038(5,63)$ \\
\hline & Pacífica & $73 / 2752(26,52)$ & $96 / 27.456(3,49)$ & $169 / 30.208(5,59)$ \\
\hline & Otros departamentos & $2 / 467(4,28)$ & $5 / 1368(3,65)$ & $7 / 1835(3,81)$ \\
\hline & Total & $520 / 25.542(20,35)$ & $483 / 166.460(2,90)$ & $1003 / 192.002(5,22)$ \\
\hline
\end{tabular}

Tabla 3. Tasa de mortalidad ajustada por edad, caracterizada por región geográfica.

\begin{tabular}{lccccccc}
\hline Región & Atlántica & Bogotá & Central & Oriental & Pacífica & Otros & Total \\
\hline Edad & & & Muertes/Total (Tasa por 1000 cirugías) & \\
\hline $18-49$ & $5 / 11.669(0,42)$ & $18 / 32.558(0,55)$ & $12 / 25.534(0,46)$ & $14 / 15.514(0,9)$ & $7 / 15.877(0,44)$ & $0 / 1274(0)$ & $56 / 102.426(0,54)$ \\
$50-64$ & $17 / 5306(3,20)$ & $56 / 18.173(3,08)$ & $45 / 13.582(3,31)$ & $26 / 7644(3,40)$ & $31 / 8503(3,64)$ & $0 / 411(0)$ & $175 / 53.619(3,26)$ \\
$65-80$ & $27 / 2508(10,76)$ & $128 / 9163(13,96)$ & $100 / 7531(13,27)$ & $74 / 3784(19,55)$ & $59 / 4497(13,11)$ & $6 / 122(49,18)$ & $394 / 27.605(14,27)$ \\
$>80$ & $38 / 678(56,04)$ & $115 / 2740(41,97)$ & $108 / 2479(43,56)$ & $44 / 1096(40,14)$ & $72 / 1331(54,09)$ & $1 / 28(35,71)$ & $378 / 8352(45,25)$ \\
Total & $87 / 20.161(4,31)$ & $317 / 62.634(5,06)$ & $265 / 49.126(5,39)$ & $158 / 28.038(5,63)$ & $169 / 30.208(5,59)$ & $7 / 1835(3,81)$ & $1003 / 192.002(5,22)$ \\
\hline
\end{tabular}

Tabla 4. Tasa de mortalidad ajustada por edad, caracterizada por sexo.

\begin{tabular}{lccc}
\hline Sexo & Masculino & Femenino & Total \\
\hline Edad (años) & \multicolumn{2}{c}{ Muertes/Total de pacientes operados (Tasa por 1000 cirugías) } \\
$18-49$ & $23 / 23.749(0,96)$ & $33 / 78.677(0,41)$ & $56 / 102.426(0,54)$ \\
$50-64$ & $93 / 16.705(5,56)$ & $82 / 36.914(2,22)$ & $175 / 53.619(3,26)$ \\
$65-80$ & $191 / 10.856(17,59)$ & $203 / 16.749(12,12)$ & $394 / 27.605(14,27)$ \\
$>80$ & $169 / 3067(55,10)$ & $208 / 5285(39,35)$ & $378 / 8352(45,25)$ \\
Total & $476 / 54.377(8,75)$ & $526 / 137.625(3,82)$ & $1003 / 192.002(5,22)$ \\
\hline
\end{tabular}


Tabla 5. Volumen quirúrgico calculado por 100.000 habitantes

\begin{tabular}{lccccccc}
\hline & Año & $\mathbf{2 0 1 2}$ & $\mathbf{2 0 1 3}$ & $\mathbf{2 0 1 4}$ & $\mathbf{2 0 1 5}$ & $\mathbf{2 0 1 6}$ & Total \\
\hline Sexo & Masculino & 132,68 & 139,90 & 107,97 & 100,46 & 110,84 & 117,95 \\
Grupo etario & Femenino & 330,27 & 340,01 & 276,04 & 250,06 & 267,78 & 291,89 \\
& $18-49$ & 176,40 & 186,29 & 148,07 & 133,70 & 147,49 & 158,21 \\
& $50-64$ & 357,05 & 361,41 & 289,62 & 260,02 & 275,35 & 306,26 \\
\multirow{4}{*}{ Región geográfica } & $65-80$ & 400,38 & 396,53 & 314,40 & 293,24 & 305,42 & 338,67 \\
& $>80$ & 404,70 & 376,51 & 275,01 & 239,13 & 227,15 & 293,13 \\
& Atlántica & 181,01 & 181,93 & 172,97 & 141,11 & 140,21 & 812,20 \\
& Bogotá D.C. & 278,32 & 284,87 & 228,28 & 187,28 & 236,46 & 1124,43 \\
& Central & 226,58 & 231,06 & 189,64 & 170,73 & 173,83 & 922,12 \\
& Oriental & 215,82 & 221,80 & 174,20 & 152,44 & 177,17 & 881,37 \\
Total & Pacífica & 238,97 & 264,13 & 171,07 & 232,17 & 195,85 & 1063,17 \\
\hline
\end{tabular}

[1] En la Base de datos para el estudio de la suficiencia de la UPC (Unidad de Pago por Capitación), se encuentran reportadas todas las atenciones en salud prestadas durante un año calendario y pagadas con recursos asociados a la UPC, información que es reportada por las EPS (Empresas Promotoras de Salud) del régimen contributivo al Ministerio de Salud Nacional de forma obligatoria. Esta base de datos contiene la siguiente información: Identificador del individuo (anonimizada), edad, sexo, EPS, fecha de atención, ciudad de la atención, tipo de atención: medicamento o procedimiento, caracterización del tipo de atención: códigos unificados para procedimientos y servicios, cantidad de medicamentos o días de estancia, diagnóstico relacionado (CIE-10), costo de la atención pagada por la EPS al prestador e identificación del prestador.

\section{Discusión}

El mayor número de casos de patología de la vía biliar ocurre en mujeres adultas ${ }^{6}$, y en este estudio el sexo femenino representó el mayor volumen quirúrgico, sin embargo, se observó que los hombres presentaron mayor mortalidad perioperatoria. Esta tasa de mortalidad aumentó significativamente en aquellos que además eran mayores de 80 años.

Con la introducción de la técnica laparoscópica para el tratamiento de la patología de la vía biliar, se ha logrado la disminución del dolor postoperatorio, de la estancia hospitalaria y de la morbimortalidad, por lo que el predominio de esta técnica ha venido en aumento en las últimas décadas a nivel mundial ${ }^{2,7}, \mathrm{y}$ Colombia no ha sido ajena a este cambio. Durante el período del estudio, se observó un llamativo contraste entre el número de personas a quienes se les realizó el procedimiento vía laparoscópica, comparado con la vía abierta, observándose en esta última una menor proporción de pacientes operados, pero una mortalidad perioperatoria notoriamente ma- yor. Queda la duda, de si, la mortalidad asociada a la técnica abierta, se debe al abordaje quirúrgico utilizado o a posibles variables confusoras individuales, no observables de los pacientes, es decir, a características individuales que están asociadas tanto al abordaje quirúrgico como al desenlace de mortalidad.

Bogotá D.C., es la ciudad donde más hospitales por habitante hay, allí se concentra gran parte de la capacidad humana, técnica y estructural del sistema de salud ${ }^{5}$. Este estudio mostró que es la ciudad con mayor volumen quirúrgico y mayor mortalidad perioperatoria total relacionada con la colecistectomía. Esto puede ser explicado porque en la ciudad se realizan los procedimientos en pacientes con mayor riesgo. Sin embargo, en la región Oriental, donde el volumen quirúrgico no es tan alto, se presentó la mayor mortalidad perioperatoria en población joven. Es importante resaltar que, en el régimen contributivo, la mayor cantidad de cirugías se realiza en personas entre los 18 y los 49 años, pero dada la proporción de afiliados por grupo 
etario, el volumen quirúrgico es mayor en las personas de 65 a 80 años.

Además, se observa que en todas las regiones del país fallecieron más los mayores de 80 años, de ambos sexos, y también por los dos abordajes quirúrgicos analizados en el estudio. Esto puede obedecer al hecho de que esta población tiende a presentar otras comorbilidades al momento de la cirugía, como se evidencia en un estudio realizado en población colombiana, en el que los mayores de 65 años tenían con mayor frecuencia condiciones como hipertensión arterial, diabetes mellitus y clasificación ASA III-IV al momento de ser llevados a colecistectomía ${ }^{8}$. Nimptsch et al., en 2015 encontraron que el riesgo de morir luego de una colecistectomía es 10 veces mayor en pacientes mayores de 65 años que en personas jóvenes ${ }^{9}$.

Al revisar la mortalidad perioperatoria asociada a colecistectomía en otros países, se encontró en un estudio retrospectivo realizado en población sueca, que el riesgo de mortalidad posteror a colecistectomía fue bajo $(0,15 \%)$, y los factores más asociados eran las complicaciones perioperatorias y la presencia de comorbilidades ${ }^{1}$. En el estudio realizado en una población alemana por Nimptsch et al., la tasa de mortalidad fue de 0,4 \%, y los factores asociados a mayor riesgo de muerte fueron ser mayor de 65 años y tener comorbilidades como falla cardiaca congestiva, enfermedad pulmonar obstructiva crónica (EPOC) y enfermedad hepática ${ }^{9}$.

McMahon et al., en el año 2000, realizaron un estudio para determinar el impacto de la colecistectomía laparoscópica para el abordaje de la patología biliar, técnica que para ese año ya era la más utilizada en Escocia, encontrando una baja mortalidad perioperatoria a los 30 días de la cirugía $(0,74 \%)$. Los factores de riesgo asociados fueron edad avanzada, sexo masculino, ingreso por urgencias y admisión previa por enfermedad cardíaca o respiratoria ${ }^{10}$. Un estudio en Estados Unidos encontró una tasa de mortalidad perioperatoria de $0,53 \%$, con mayor incidencia en colecistectomías abiertas ${ }^{11}$. Nuestro estudio encontró una mortalidad perioperatoria de $0,52 \%$, resultado similar a los estudios antes mencionados.
Un estudio realizado por Bray et al. ${ }^{12}$, encontró un volumen de colecistectomías de 182,6 por 100.000 habitantes en Francia, en un periodo de 7 años, mientras que en Suecia se estimó una media de 157 cirugías por 100.000 habitantes en un periodo de 5 años ${ }^{13}$. El volumen quirúrgico hallado en este estudio en los 5 años estimados fue de 206 colecistectomías por 100.000 afiliados, cifra que comparada con los dos estudios mencionados anteriormente, es mayor.

La principal limitante de este estudio fue que solo se contó con datos de afiliados al régimen contributivo de salud, lo que representa alrededor del $46 \%$ de los afiliados al Sistema General de Seguridad Social en Salud (SGSSS) en el año 2013 5 . Aun así, la población incluida es extrapolable al régimen subsidiado para los objetivos del estudio.

Como lo describe la Lancet Commission on Global Surgery 2030: "La cirugía es una parte indispensable del sistema de salud. Prevenir y tratar las condiciones quirúrgicas es necesario para mejorar la salud de la población, es una parte fundamental en un sistema de salud resiliente y es una parte crucial para lograr los objetivos globales en salud" 3 , por tal razón, es importante invertir esfuerzo en estimar estadísticas poblacionales de cuidado quirúrgico. Se espera que este estudio sea tenido en cuenta para determinar estadísticas nacionales oficiales de mortalidad perioperatoria y volumen quirúrgico, de las patologías que son fácilmente tratables y prevenibles, y que, a partir de estas, se determinen las metas de mortalidad para 2030, así como los esfuerzos por parte de entes nacionales para lograr el objetivo de 5000 cirugías por 100.000 habitantes.

\section{Conclusiones}

En este estudio la tasa de mortalidad perioperatoria para colecistectomía fue baja, y se encontró mayor mortalidad en la población mayor de 80 años, de sexo masculino, en cirugía vía abierta o por laparotomía, así como en operados en la ciudad de Bogotá, D.C. El volumen quirúrgico estimado en este estudio fue similar o superior al encontrado en estudios realizados en otras poblaciones, y fue superior en el sexo femeni- 
no, en población entre los 65 y los 80 años, y en Bogotá D.C.

Este estudio espera ser un precedente para investigar a fondo factores de riesgo y de calidad de la atención quirúrgica en la población colombiana, para contar con cifras oficiales de mortalidad y volumen quirúrgico de colecistectomía, así como para avanzar en las metas propuestas por Lancet Commission on Global Surgery 2030.

\section{Consideraciones éticas}

Consentimiento informado: Este trabajo se base en la revisión de una base de datos, por lo que no se requiere consentimiento informado por parte de los pacientes. El presente estudio fue aprobado por el Comité de Ética de la Facultad de Medicina de la Universidad Nacional de Colombia.

Conflictos de interés: Los autores del presente estudio declaran no tener conflictos de interés.

Financiación: Debido a que las bases de datos utilizadas son de acceso público, no hubo financiación de terceros para el desarrollo de este estudio.

Agradecimientos: A la Universidad Nacional de Colombia por darnos herramientas y espacios para investigación, al Instituto de Investigaciones Clínicas de la Facultad de Medicina y a Vitruvio, Anatomía Clínica y Quirúrgica.

Contribución de los autores: Análisis e interpretación de datos, redacción y revisión del artículo: Daniela Escalante-Arbeláez y Marcela Bernal-Gutiérrez.

Diseño del estudio, recolección de la información, análisis e interpretación de datos, redacción y revisión del artículo: Giancarlo Buitrago.

\section{Referencias}

1. Sandblom G, Videhult P, Crona Guterstam Y, Svenner A, SadrAzodi O. Mortality after a cholecystectomy: a population-based study. HPB. 2014;17(3):239-243. https://doi.org/10.1111/hpb.12356

2. Scollay JM, Mullen R, McPhillips G, Thompson AM. Mortality associated with the treatment of gallstone disease: A 10-year contemporary national experience. World J Surg. 2010;35(3):643-647. https://doi.org/10.1007/s00268-010-0908-3

3. Meara JG, Leather A, Hagander L, Alkire BC, Alonso N, Ameh ES, et al. Global Surgery 2030: Evidence and solutions for achieving health, welfare, and economic development. The Lancet. 2015;386:569-624. https://doi.org/10.1016/S0140-6736(15)60160-X.

4. Shaffer EA. Epidemiology and risk factors for gallstone disease: Has the paradigm changed in the 21st century?. Current Gastroenterol Rep. 2005;7:12-40. https://doi.org/10.1007/s11894-005-0051-8

5. Guzmán, K. Radiografía de la oferta de servicios de salud en Colombia. Documentos de trabajo sobre economía regional. Banco de la República. Centro de estudios económicos regionales (CEER): Cartagena; 2017. https://www.banrep.gov.co/sites/default/files/ publicaciones/archivos/dtser_202.pdf.

6. Steiner CA. Bass EB, Talamini MA, Pitt HA, Steinberg EP. Surgical rates and operative mortality for open and laparoscopic cholecystectomy in Maryland. N Engl J Med. 1994;330:403-8. https://doi.org/10.1056/NEJM199402103300607

7. Rosenmüller M, Haapamäki, M, Nordin P, Stenlund $H$, Nilsson E. Cholecystectomy in Sweden 2000-2003: A nationwide study on procedures, patient characteristics, and mortality. BMC Gastroenterol. 2007;7:35-7. https://doi:10.1186/1471-230X-7-35

8. Domínguez LC, Herrera WE, Rivera AM, Bermúdez CE. Colecistectomía de urgencia por laparoscopia por colecistitis aguda en adultos mayores. Rev Colomb Cir. 2011;26:93-100.

9. Nimptsch U, Mansk T. Deaths following cholecystectomy and herniotomy: An analysis of nationwide german hospital discharge data from 2009 to 2013. Dtsch Arztebl Int. 2015;112:535-43.

https://doi.org/10.3238/arztebl.2015.0535

10. McMahon AJ, Fischbacher CM, Frame SH, MacLeod MC. Impact of laparoscopic cholecystectomy: A population-based study. Lancet. 2000;(356):1632-7. https://doi.org/10.1016/s0140-6736(00)03156-1

11. Ingraham AM, Cohen ME, Ko CY, Hall BL. A current profile and assessment of North American cholecystectomy: Results from the American College of Surgeons National Surgical Quality Improvement Program. J Am Coll Surg. 2010;211:176-86. https://doi:10.1016/j.jamcollsurg.2010.04.003.

12. Bray F, Balcaen T, Baro E, Gandon A, Ficheur G, Chazard E. Increased incidence of cholecystectomy related to gallbladder disease in France: Analysis of 807,307 cholecystectomy procedures over a period of seven years. J Visc Surg. 2019;156:209-15. https://doi.org/10.1016/j.jviscsurg.2018.12.003.

13. Noel R, Arnelo U, Enochsson L, Lundell L, Nilsson M, Sandblom G. Regional variations in cholecystectomy rates in Sweden: impact on complications of gallstone disease. Scand J Gastroenterol. 2016;51:465-71. https://doi.org/10.3109/00365521.2015.1111935 